\title{
Electronic Data Capture Activation
}

National Cancer Institute

\section{Source}

National Cancer Institute. Electronic Data Capture Activation. NCI Thesaurus. Code C142528.

The act or process of enabling an electronic data capture system to acquire data. 